\title{
AQUISIÇÃO DOS FONEMAS FRICATIVOS CORONAIS POR CRIANÇAS DA REGIÃO METROPOLITANA DO RECIFE
}

\author{
The acquisition of coronal fricatives by children \\ of Recife's metropolitan region
}

\author{
Ana Augusta de Andrade Cordeiro (1), Jakelline Miranda Alves (2), \\ Bianca Arruda Manchester de Queiroga ${ }^{(3)}$, Ana Cristina Montenegro ${ }^{(4)}$, \\ Stela Telles ${ }^{(5)}$, Rafaella Asfora ${ }^{(6)}$
}

\section{RESUMO}

Objetivo: investigar a aquisição das fricativas coronais por crianças de creches/escolas públicas da Região Metropolitana do Recife. Métodos: 40 crianças frequentadoras de quatro creches/escolas, com idades entre 2 a 6 anos e 11 meses, sendo 20 do sexo masculino e 20 do sexo feminino. $O$ instrumento utilizado foi constituído por figuras que visavam à nomeação de 83 palavras que possuíam os fonemas-alvos: /s/ (32), /z/ (11), / / / (21), /3/ (19), selecionadas a partir das variáveis linguísticas tonicidade e estrutura silábica. As crianças foram divididas em 10 grupos, de acordo com a faixa etária. Considerou-se o percentual de $80 \%$ de produção como indicativo de aquisição do fonemaalvo. Resultados: o início da produção das fricativas coronais deu-se na faixa etária de 2:0-2:5 anos. Observou-se que os fonemas $/ \mathrm{J} /, / 3 / \mathrm{e} / \mathrm{z} /$ foram adquiridos aos 3:0-3:5 anos e o fonema $/ \mathrm{s} /$ aos 2:62:11 anos. Os processos fonológicos mais comumente encontrados foram substituição e omissão. Também foi observado com bastante frequência a troca semântica. Conclusões: a aquisição das fricativas coronais das crianças frequentadoras de escolas e creches públicas da Região Metropolitana do Recife ocorre mais tardiamente quando comparada à literatura nacional e sofre influência de variáveis linguísticas. É importante salientar que há uma diversidade linguística e sócio-cultural no território brasileiro, de forma que os dados observados em algumas regiões não são necessariamente correspondentes em todas as regiões do Brasil. Esse aspecto deve ser considerado em estudos que pretendem fixar parâmetros de avaliação fonológica, sobretudo se houver fins de diagnóstico de desvios fonológicos.

DESCRITORES: Estudos de Linguagem; Desenvolvimento da Linguagem; Fonética

(1) Fonoaudióloga; Professora do Departamento de Fonoaudiologia da Universidade Federal de Pernambuco, UFPE, Recife, PE, Brasil; Doutora em Psicologia pela Universidade Federal de Pernambuco.

(2) Fonoaudióloga; Bolsista de fixação de Técnico em Pesquisa pela Fundação de Amparo à Ciência e Tecnologia do Estado de Pernambuco, FACEPE.

(3) Fonoaudióloga; Professora do Departamento de Fonoaudiologia da Universidade Federal de Pernambuco, UFPE, Recife, PE, Brasil; Doutora em Psicologia pela Universidade Federal de Pernambuco.

(4) Fonoaudióloga do Serviço Assistência Social ao Servidor da Policia Civil, Recife, PE, Brasil; Doutoranda em Linguística na Universidade Federal de Pernambuco.

(5) Linguista; Professora do Departamento de Letras do Programa de Pós-Graduação em Letras (na área de concentração em Linguística) da Universidade Federal de

\section{INTRODUÇÃO}

O desenvolvimento fonológico é um processo complexo ${ }^{1}$ que ocorre de forma não linear ${ }^{2}$ e com variações individuais, necessitando do amadurecimento do conhecimento fonológico num processo gradativo $^{3}$. Como resultado deste desenvolvimento,

Pernambuco, UFPE, Recife, PE, Brasil; Doutora em Linguista pela Vrije Universiteit.

(6) Fonoaudióloga; Professora do Departamento de Educação da Universidade Federal de Pernambuco, UFPE, Recife, PE, Brasil; Doutora em Psicologia pela Universidade Federal de Pernambuco.

Conflito de interesses: inexistente 
tem-se o estabelecimento de um sistema condizente com o padrão adulto, que é a fala do grupo social em que a criança está inserida ${ }^{4}$

Nos últimos anos, observa-se no Brasil grande interesse por parte de pesquisadores em colaborar para uma maior compreensão do desenvolvimento fonológico do português brasileiro. Entretanto, a maioria dos estudos conduzidos até o momento concentra-se nas regiões Sul e Sudeste do país ${ }^{5}$ e é importante salientar que, em virtude da dimensão continental do Brasil e da heterogeneidade social, há uma diversidade de variações linguísticas em todo território brasileiro ${ }^{6}$.

Assim, apesar de haver um aumento de pesquisas em aquisição fonológica nas últimas décadas ${ }^{4,7,8}$, observa-se uma carência de estudos que explorem a aquisição fonológica em outras regiões do país, especialmente na região Nordeste.

Algumas pesquisas em aquisição fonológica estão relacionadas à prática fonoaudiológica ${ }^{2,9}$, auxiliando no diagnóstico e no tratamento dos desvios fonológicos ${ }^{3,7}$.

Um estudo pioneiro, realizado na região Sul sobre a aquisição fonológica do português, em $1990^{10}$, observou que o /s/ em onset inicial está dominado aos 2:2 anos, em onset medial aos 2:10 anos. O fonema / $z /$ está dominado em onset medial aos 2:6 e em onset inicial aos 3:1. Os fonemas / $/ \mathrm{e}$ /3/ estão plenamente adquiridos nas duas posições somente aos 4:0 anos.

De modo um pouco diferente, outros estudos realizados nas regiões Sul e Sudeste do país, o domínio das fricativas coronais ocorre em torno dos 3:0 anos de idade ${ }^{5,7}$. Entretanto, esta aquisição não acontece de modo linear ${ }^{4}$. Os primeiros sons surgem por volta de 1:3 anos, sendo o fonema sonoro adquirido anteriormente ao surdo ${ }^{7,11}$. De acordo com tais pesquisas, os fonemas sonoros |z/ e /3/ são adquiridos aos 2:0 e 2:6 anos e o /s/ e / / / aos 2:6 e 2:10 anos ${ }^{7}$. Tais achados diferem de outros estudos realizados em $2006^{12}$ e $2008^{13}$ que afirmam que o fonema /s/ é produzido anteriormente ao /z/.

Dentre as variáveis linguísticas que estão relacionadas ao percurso de aquisição das fricativas coronais, destacam-se a posição do onset na palavra $^{7,10,11}$ e a tonicidade; observando-se com mais frequência os processos fonológicos de substituição e de omissão ${ }^{4,7}$.

Uma pesquisa realizada em $2001^{11}$ sobre a aquisição das fricativas envolvendo apenas /s/ e /z/, a partir de dois bancos de dados, considerou variáveis linguísticas e extralinguísticas e concluiu que, quando não há produção adequada do segmento para o /z/, há uma preferência pela substituição por [3], [s] e []]. Quanto ao fonema /s/, a substituição preferida é por [J] e, menos frequentemente, por [ $\mathrm{t}]$ e $\left[\mathrm{t} \int\right]$.

Considerando que os fonemas fricativos coronais (/f/ e /z/, /s/, /z/) são de grande importância para a comunicação, que apresentam frequentes alterações ${ }^{7,11}$ e que há uma carência de estudos conduzidos na região Nordeste, esta pesquisa estudará a aquisição desses fonemas em posição de onset silábico.

O objetivo do presente estudo foi, portanto, investigar a aquisição das fricativas coronais por crianças com idade entre 2:0 a 6:11 anos de creches/escolas públicas municipais da Região Metropolitana do Recife.

Espera-se que o estudo possa auxiliar no mapeamento da aquisição fonológica da região Nordeste, oferecendo subsídios aos pais, professores, linguistas, fonoaudiólogos e demais profissionais envolvidos com o estudo do desenvolvimento fonológico infantil, para que possam diferenciar variação linguística de desvio fonético e fonológico.

\section{MÉTODOS}

Participaram do estudo 40 crianças pertencentes à faixa etária de 2 a 6:11 anos, sendo 20 meninos e 20 meninas distribuídas em 10 grupos etários: G1 (2 - 2:5 anos); G2 (2:6 - 2:11anos); G3 (3 - 3:5 anos); G4 (3:6 - 3:11 anos); G5 (4 - 4:5 anos); G6 (4:6 - 4:11 anos); G7 (5 - 5:5 anos); G8 (5:6 - 5:11anos); G9 (6 - 6:5 anos); G10 (6:6 - 6:11 anos).

Além da idade e do fato de estarem matriculadas em creches e escolas públicas, para serem incluídas na amostra as crianças deveriam ser indicadas pelos professores, com base no critério de serem crianças comunicativas e não apresentarem características de distúrbios da comunicação.

Como variáveis de estudo foram consideradas, além da idade (2:0 a 6:11 anos) e do gênero (sexo masculino e feminino), as variáveis linguísticas: tonicidade (sílaba tônica, pré-tônica e pós-tônica) e posição silábica na palavra (onset inicial e onset medial).

Antes de se iniciar a coleta dos dados, todos os responsáveis pelos participantes receberam uma carta de informação, a qual continha os objetivos do estudo e a garantia da não identificação dos mesmos. Os que concordaram em participar da pesquisa assinaram o Termo de Consentimento Livre e Esclarecido. Em seguida, foram realizados os seguintes procedimentos:

a) Avaliação fonoarticulatória: foram examinadas estrutura, tonicidade, e mobilidade dos órgãos fonoarticulatórios das crianças. Seu objetivo 
foi assegurar a participação de crianças sem alterações desta ordem.

b) Avaliação fonológica: foi utilizada a Prova de Avaliação Fonológica -PAFON, um instrumento em fase de validação, que inclui prova de nomeação espontânea de 215 figuras, selecionadas com base em critérios linguísticos de modo a controlar a influência de aspectos como tonicidade e posição silábica dos fonemas.

Para efeito de análise, elegeu-se 83 figuras. Dentre estas figuras, 32 objetivavam a produção do fonema /s/, 11 a produção do fonema /z/, 21 a produção do fonema $/ /$ / e 19 a produção do fonema $/ 3 /$.

A gravação da produção oral, individual, foi realizada com gravador digital da marca Olympus modelo VN-4100PC, e as respostas foram transcritas de acordo com o IPA (International Phonetic Alphabet).

A presente pesquisa faz parte de um projeto maior denominado "Aquisição fonológica do português não-padrão falado na região metropolitana do Recife", que foi aprovado pelo Comitê de Ética do Centro de Ciências da Saúde (CCS) da Universidade Federal de Pernambuco sob o protocolo № $075 / 06$, tendo seguido todos os preceitos éticos preconizados na Resolução 196/96 do CNS.

Os dados foram submetidos à análise de estatística descritiva, sendo utilizado o critério de $80 \%$ de produção para indicar a aquisição do fonema.

\section{RESULTADOS}

Como mencionado anteriormente, para efeito de análise, elegeu-se a nomeação de 83 figuras. Dentre estas figuras, 32 objetivavam a produção do fonema /s/, 11 a produção do fonema /z/, 21 a produção do fonema / / e 19 a produção do fonema /3/, tanto em onset inicial quanto medial e em posições pré-tônicas, tônicas e pos-tônicas. O objetivo da análise foi identificar o início da produção do som nestes diferentes contextos lingüísticos e sua aquisição na população estudada.

Tabela 1 - Distribuição do surgimento e aquisição dos fonemas fricativos coronais na população estudada nos grupos etários de acordo com posição silábica das palavras que possuem o fonema

\begin{tabular}{|c|c|c|c|c|c|}
\hline Onset & Tonicidade & Fonema /s/ & Fonema /z/ & Fonema $/ \mathrm{j} /$ & Fonema /3/ \\
\hline \multirow{2}{*}{ Inicial } & Pré-tônica & $2: 0-2: 5 / 3: 0-3: 5$ & $\begin{array}{c}2: 6-2: 11 / 3: 0- \\
3: 5\end{array}$ & $2: 0-2: 5 / 3: 0-3: 5$ & $2: 0-2: 5 / 3: 0-3: 5$ \\
\hline & Tônica & $2: 0-2: 5 / 3: 0-3: 5$ & $2: 0-2: 5 / 3: 0-3: 5$ & $2: 0-2: 5 / 3: 0-3: 5$ & $2: 0-2: 5 / 3: 0-3: 5$ \\
\hline \multirow{3}{*}{ Medial } & Pré-tônica & $\begin{array}{c}2: 0-2: 5 / 2: 6- \\
2: 11\end{array}$ & - & - & - \\
\hline & Tônica & $\begin{array}{c}2: 0-2: 5 / 2: 6- \\
2: 11\end{array}$ & $2: 0-2: 5 / 3: 0-3: 5$ & $2: 0-2: 5 / 3: 0-3: 5$ & $2: 0-2: 5 / 3: 0-3: 5$ \\
\hline & Pós-tônica & $\begin{array}{c}2: 0-2: 5 / 2: 6- \\
2: 11\end{array}$ & $2: 0-2: 5 / 3: 0-3: 5$ & $2: 0-2: 5 / 3: 0-3: 5$ & $2: 0-2: 5 / 3: 0-3: 5$ \\
\hline
\end{tabular}

Como é possível observar na Tabela 1, o início da produção das fricativas coronais em onset inicial, em sílaba pré-tônica e tônica, ocorreu na faixa de 2:0-2:5 anos para todos os fonemas, com exceção do fonema /z/ que, em sílaba pré-tônica, surgiu aos 2:6-2:11 anos. Quanto à aquisição foi observado que esta ocorre aos 3:0-3:5 anos.

Já em onset medial, em sílaba pré-tônica, tônica e pós-tônica o início de produção aconteceu na faixa de 2:0-2:5 anos e aquisição aos 3:0-3:5 anos, com exceção do fonema /s/ que adquiriu estabilidade mais cedo (aos 2:6-2:11 anos), independentemente da tonicidade.

É possível perceber, portanto, a influência da posição silábica sobre a aquisição, uma vez que a estabilidade ocorreu aos 2:6-2:11 anos em onset medial e aos 3:0-3:5 anos em onset inicial.

Durante a aquisição fonológica, além das produções típicas (padrão) é comum a presença de processos fonológicos operando nessa aquisição. Por esta razão, a seguir são apresentados os percentuais de produção padrão e dos processos fonológicos observados na aquisição dos fonemas estudados.

Além disso, considerando a aplicação do instrumento de avaliação (prova de nomeação), algumas crianças não reconheceram a figura e não realizaram a produção das palavras-alvo, ou realizaram uma troca semântica, o que, em ambos os casos, não permitia a realização das análises. Por este motivo, os valores percentuais de "não produção" também estão apresentados nos resultados. 
Tabela 2 - Percentuais de produção padrão e estratégias de reparo observados na aquisição do /s/, considerando onset e tonicidade

\begin{tabular}{|c|c|c|c|c|c|}
\hline Onset & Tonicidade & Padrão & Substituição & Omissão & Não produziu \\
\hline \multirow{5}{*}{ Inicial } & Pré-tônica & & & & \\
\hline & 06 palavras & $78,75 \%$ & $9,17 \%$ & $1,25 \%$ & $10,83 \%$ \\
\hline & 240 produções & & & & \\
\hline & Tônica & & & & \\
\hline & $\begin{array}{c}05 \text { palavras } \\
200 \text { producoões }\end{array}$ & $79,5 \%$ & $10,5 \%$ & ---- & $10 \%$ \\
\hline \multirow{9}{*}{ Medial } & Pré-tônica & & & & \\
\hline & 02 palavras & $75 \%$ & $10 \%$ & $3,75 \%$ & $11,25 \%$ \\
\hline & 80 produções & & & & \\
\hline & Tônica & & & & \\
\hline & 09 palavras & $78,61 \%$ & $11,39 \%$ & ---- & $10 \%$ \\
\hline & 360 produções & & & & \\
\hline & Pós-tônica & & & & \\
\hline & 10 palavras & $77,25 \%$ & $11 \%$ & $0,25 \%$ & $11,5 \%$ \\
\hline & 400 produções & & & & \\
\hline
\end{tabular}

Como é possível observar na Tabela 2, na aquisição do /s/ foram observados os processos fonológicos de substituição, em torno de $10 \%$ em todas as posições, seguido de omissão, com percentuais bem mais baixos, variando de $0,25 \%$ a $3,75 \%$. É importante observar que a omissão não ocorreu em sílabas tônicas.

Tabela 3 - Percentuais de produção padrão e estratégias de reparo observados na aquisição do /z/, considerando onset e tonicidade

\begin{tabular}{|c|c|c|c|c|c|}
\hline Onset & Tonicidade & Padrão & Substituição & Omissão & Não Produziu \\
\hline \multirow{5}{*}{ Inicial } & Pré-tônica & & & & \\
\hline & 01 Palavra & $57,5 \%$ & $25 \%$ & $7,5 \%$ & $10 \%$ \\
\hline & 40 Produções & & & & \\
\hline & Tônica & & & & \\
\hline & $\begin{array}{l}02 \text { Palavras } \\
80 \text { Produções }\end{array}$ & $81,25 \%$ & $6,25 \%$ & $1,25 \%$ & $11,25 \%$ \\
\hline \multirow{6}{*}{ Medial } & Tônica & & & & \\
\hline & 05 palavras & $76,5 \%$ & $10,5 \%$ & ---- & $13 \%$ \\
\hline & 200 produções & & & & \\
\hline & Pós-tônica & & & & \\
\hline & 03 palavras & $82,5 \%$ & $8,3 \%$ & ---- & $9,17 \%$ \\
\hline & 120 Produções & & & & \\
\hline
\end{tabular}

No caso do fonema /z/, também se verificou que os processos mais frequentes foram substituição e omissão. No entanto, de modo diferente do que foi observado no /s/, percebe-se um maior percentual de substituições (chegando a $25 \%$ em sílaba prétônica) e não foram observadas omissões quando o fonema estava em posição de onset medial. 
Tabela 4 - Percentuais de produção padrão e estratégias de reparo observados na aquisição do IJ/,considerando onset e tonicidade

\begin{tabular}{|c|c|c|c|c|c|}
\hline Onset & Tonicidade & Padrão & Substituição & Omissão & Não Produziu \\
\hline \multirow{4}{*}{ Inicial } & Pré-tônica & & & & \\
\hline & $\begin{array}{c}7 \text { palavras } \\
280 \text { producões }\end{array}$ & $80 \%$ & $7,92 \%$ & $1,25 \%$ & $10,83 \%$ \\
\hline & Tônica & & & & \\
\hline & $\begin{array}{c}3 \text { palavras } \\
120 \text { produções }\end{array}$ & $75,83 \%$ & $5,83 \%$ & $0,83 \%$ & $17,5 \%$ \\
\hline \multirow{4}{*}{ Medial } & Tônica & & & & \\
\hline & 3 palavras & 80,83 & $7,5 \%$ & 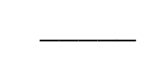 & $10,83 \%$ \\
\hline & $\begin{array}{l}\text { Pós-tônica } \\
\text { Proauçoes }\end{array}$ & & & & \\
\hline & $\begin{array}{c}8 \text { palavras } \\
320 \text { produções }\end{array}$ & $80,62 \%$ & $9,69 \%$ & - & $9,69 \%$ \\
\hline
\end{tabular}

Em relação aos processos de produção do $/ \mathrm{f} /$, também foi observada a ocorrência do processo de substituição, seguido de omissão. No caso da substituição, os percentuais variaram de $5,83 \%$ a 9,69\%. Já o processo de omissão só ocorreu em onset inicial, tanto em sílaba pré-tônica como em sílaba tônica, porém com percentuais muito baixos de $0,83 \%$ a $1,25 \%$. Em onset medial, o processo de omissão não ocorreu.

Tabela 5 - Percentuais de produção padrão e estratégias de reparo observados na aquisição do /3/, considerando onset e tonicidade

\begin{tabular}{|c|c|c|c|c|c|}
\hline Onset & Tonicidade & Padrão & Substituição & Omissão & Não Produziu \\
\hline \multirow{6}{*}{ Inicial } & Pré-tônica & & & & \\
\hline & 7 palavras & 78,21 & 9,64 & & 11,43 \\
\hline & 280 produções & & & & \\
\hline & Tônica & & & & \\
\hline & 1 palavras & $80 \%$ & $7,5 \%$ & & $12,5 \%$ \\
\hline & 40 produções & & & & \\
\hline \multirow{6}{*}{ Medial } & Tônica & & & & \\
\hline & 5 palavras & $74,5 \%$ & $13 \%$ & 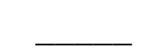 & $12,5 \%$ \\
\hline & 200 produções & & & & \\
\hline & Pós-tônica & & & & \\
\hline & 6 palavras & $78,44 \%$ & $10 \%$ & $\bar{\square}$ & $10,0 \%$ \\
\hline & 240 produções & & & & \\
\hline
\end{tabular}

Como se observa na Tabela, com relação à produção do /3/ foi observado apenas o processo de substituição, com percentuais variando entre $7,5 \%$ a $13 \% \%$. Não foi observado o processo de omissão independentemente do onset e da tonicidade.

É interessante observar que, tanto no caso do fonema /z/, quanto no /3/, não foi constatado 0 processo de ensurdecimento das fricativas sonoras que se apresenta frequente em crianças com distúrbio fonológico ${ }^{10}$.

\section{DISCUSSÃO}

Estudos que investigaram a aquisição das fricativas coronais afirmam que estes fonemas estão adquiridos no sistema fonológico das crianças até os 3:0 anos ${ }^{5}$, com muita variabilidade entre eles e conforme a posição que ocupam na palavra e na sílaba ${ }^{7,11}$ Assim, um paralelo entre os resultados encontrados nas crianças da presente pesquisa 
e a literatura nacional sobre a aquisição das fricativas coronais permite a observação de diferenças importantes, mesmo em estudos conduzidos numa mesma região geográfica.

Na região Sul do Brasil, considerando a posição do fonema na palavra para efeito de analise, em relação ao fonema /s/, por exemplo, uma parte da literatura ${ }^{7}$ afirma que a aquisição $0 / \mathrm{s} / \mathrm{se}$ dar aos 2:0 anos em posição de onset medial, e aos 2:6 anos em posição de onset inicial. Outro estudo ${ }^{12}$ realizado na região Sudeste em 2006 indica uma aquisição mais tardia para a aquisição do seguimento, apontando as idades de 3:1 anos em onset medial e 3:2 anos em onset inicial. De modo diferente, estudos também realizados nas regiões Sul, em $1990^{10}$ e Sudeste, em $2008^{13}$ referem que o /s/ é adquirido primeiro em onset inicial, sendo adquirido no Sul aos 2:2 anos, em onset inicial, e aos 2:10, em onset medial.Já no Sudeste o estudo aponta para as idades de 2:4 anos, em onset inicial, e 2:5 anos, em onset medial.

$\mathrm{Na}$ presente pesquisa, corroborando o estudo que também considerou a posição do fonema na palavra ${ }^{11,12}$, foi observado que a posição silábica interfere na aquisição do fonema /s/, uma vez que o som foi adquirido na faixa etária de 2:6-2:11 anos apenas no contexto do onset medial, já que em onset inicial esta aquisição só foi observada na faixa de 3:0-3:5 anos.

Quanto à tonicidade, estudo realizado na região Sul ${ }^{11}$ constatou a sílaba pós-tônica como favorecedora para a produção do /s/. Neste estudo não foi observada a influência da tonicidade na produção do /s/.

Tomados em conjunto esses resultados revelam a falta de consenso entre os estudos quanto à faixa etária em que ocorre a estabilidade na aquisição do fonema /s/. Tais diferenças não podem ser explicadas apenas pela variação regional, visto que estão descritos resultados diferentes para pesquisas conduzidas em uma única região. Além disso, é importante salientar que, na fala adulta, a produção do fonema $/ \mathrm{s} /$, na posição de onset não apresenta grandes variações regionais, mas apenas na posição de coda é que o fonema apresenta larga variação regional.

Quanto ao início de produção do fonema /s/, a literatura ${ }^{12}$ sugere que as crianças iniciam a produção do fonema aos 1:6 anos em onset inicial e aos $1: 7$ anos em onset medial. Todavia, no presente estudo o início de produção foi observado a partir da faixa etária de 2:0 anos independente do onset. No entanto, é importante destacar que a presente pesquisa investigou crianças a partir de 2 anos, não havendo dados anteriores a essa faixa de idade. Desse modo, comparações quanto ao início da produção, neste caso, não é viável, devendo futuros estudos incluir crianças menores de 2 anos.

No que se refere ao fonema $/ z /$, um estudo realizado na região Sul do país ${ }^{10}$ afirma que o fonema /z/ é adquirido primeiro em onset medial, aos 2:6 anos, e depois em onset inicial, aos 3:1 anos. Outros estudos, também realizados na região Sul, referem que a aquisição do /z/ acontece entre 2:0 anos ${ }^{11}$, e 2:2 anos ${ }^{13}$. Em um estudo ${ }^{12}$ longitudinal realizado na região Sudeste com uma criança, compreendida na faixa etária de 1:0-3:9 anos, não foi observada a presença do /z/ em onset inicial, porém em onset medial sua aquisição foi observada aos 3:1 anos.

No presente estudo foi verificado que a aquisição, tanto em onset inicial quanto medial ocorreu na faixa etária de 3:0-3:5 anos, portanto, um pouco mais tardia que o descrito nos estudos acima, sobretudo no estudo que considerou a posição do fonema na palavra ${ }^{10}$

Quanto ao início de produção do fonema /z/, um estudo realizado na região Sudeste, em $2006^{12}$ sugere que as crianças iniciam sua produção aos $1: 8$ anos em onset medial. Outro estudo também realizado na região Sudeste, em $2008^{13}$, afirma que as crianças iniciam sua produção aos 2:2 anos em onset medial. Vale ressaltar que em nenhuma destas pesquisas as crianças produziram o fonema /z/ em onset inicial. Entretanto, no presente estudo, o início de produção foi observado a partir da faixa etária de 2:0 anos, independente do onset.

Interessante destacar que o instrumento utilizado para avaliação fonológica na presente pesquisa possui apenas uma palavra para 0 fonema /z/ no contexto de onset inicial + sílaba prétônica, a palavra "zoológico" e que, nesse contexto, a produção iniciou mais tardiamente, na faixa etária de 2:6-2:11 anos, mas também teve sua aquisição confirmada aos 3:0-3:5 anos. Esta palavra poderia gerar uma produção mais tardia uma vez que as palavras polissílabas são mais raras no léxico infantil, além de que, as omissões do segmento ou da sílaba que possui o segmento ocorrem com mais frequência em posição pré-tônica.

Quanto à tonicidade, um estudo realizado na região Sul ${ }^{11}$ constatou a sílaba pré-tônica como favorecedora para a produção do /z/. Neste estudo o início da produção se deu mais tardiamente quando se tratava de sílaba pré-tônica.

Mais uma vez, destaca-se a falta de consenso da literatura a cerca dos parâmetros de aquisição fonológica.

Em relação ao fonema $/ \mathrm{f} /$, estudos $^{7}$ conduzidos na região Sul referem que sua aquisição não ocorre de forma linear. $O$ início da produção ocorre aos 1:3 anos e que seria estabilizada aos 2:10 anos. Referem que o onset medial é o mais favorecedor 
à produção desta fricativa. Referem, ainda, que o / / / está adquirido a partir de 3:6 anos na posição de onset inicial e aos 2:10 anos na posição de onset medial.

Outro estudo ${ }^{12}$ realizado na região Sudeste refere que o início de produção do / $/$ ocorre aos $1: 3$ anos e sua estabilidade se dar aos $1: 9$ anos, estando em consonância com outros estudos realizados no Sul do país, quando referem que o onset medial é favorecedor a produção desta fricativa, pois em onset medial está adquirido aos 1:8 anos e em onset inicial aos 1:9 anos.

Quanto à tonicidade, um estudo realizado na região $\mathrm{Sul}^{7}$ constatou a sílaba pós-tônica como favorecedora para a produção do $/ \mathrm{J} /$, seguida da sílaba tônica e como menos favorecedora, a prétônica. Neste estudo não foi observada a influência da tonicidade na produção do $/ \mathrm{f} /$.

Com uma diferença de pelo menos seis meses, na presente investigação, o início de produção ocorreu na faixa etária de 2:0-2:5 anos, atingindo aquisição na faixa etária de 3:0-3:5 anos. Porém, conforme já foi apontado, não é possível confrontar os resultados da presente pesquisa com os da literatura no que se refere à idade de início de produção, visto que não foram investigadas crianças com idades inferiores a dois anos.

Porém, em outro estudo realizado na região Sul, em $1990^{10}$, há evidências de aquisição mais tardia (4:0 anos).

Em relação ao fonema $/ 3 /$, o início de sua produção ocorreu na faixa etária de 2:0-2:5 anos, atingindo sua aquisição na faixa etária de 3:0-3:5 anos, diferindo de estudos realizados na região $\mathrm{Sul}^{7},{ }^{11} \mathrm{em}$ que o início da produção foi verificado aos 1:6 anos e aquisição aos 2:6 anos, portanto, mais cedo do que na população estudada na presente investigação.

Estudo realizado no $\mathrm{Sul}^{7}$ refere que o onset inicial mostrou-se mais favorecedor à produção do fonema do que o onset medial. Em posição de onset inicial, o / $/ 3$ encontra-se adquirido a partir de 2:6 anos e na posição do onset medial, só é adquirido aos 3:6 anos, ou seja, um ano depois.

Em um estudo longitudinal ${ }^{12}$, realizado na região Sudeste, com uma criança compreendida na faixa etária de 1:0-3:9 anos, não foi observada a presença do $/ 3 /$ em onset medial, porém em onset inicial sua estabilidade ocorre aos 2:2 anos.

No entanto, a literatura também aponta evidências de estabilidade mais tardia. Em estudo realizado no Sul, o /3/ nas duas posições somente encontra-se adquirido aos 4:0 anos ${ }^{10}$, diferindo de pesquisa realizada no Sudeste, em $2006^{12}$, em que o fonema $/ 3 /$ está plenamente adquirido aos 2:2 anos.
Quanto à tonicidade, estudo realizado na região $\mathrm{Sul}^{7}$ constatou a sílaba pré-tônica como favorecedora para a produção do /3/. Neste estudo não foi observada a influência da tonicidade na produção do $/ 3 /$.

Em relação aos processos fonológicos mais encontrados nas crianças na fase de aquisição dos fonemas fricativos coronais, foram observados: substituição (envolvendo traço contínuo), e omissão.

Quando examinados os fonemas em particular, algumas diferenças foram encontradas. No caso do fonema /s/ ocorreram omissões de sílaba prétônicas tanto em onset inicial quanto em onset medial, confirmando os estudos na região Sull1 , além de omissão em sílaba pós-tônica.

No caso das substituições, o fonema /s/ foi preferencialmente substituído por/t/, na qual a fricativa é produzida como uma plosiva, havendo uma mudança no traço contínuo. Também ocorreram substituições por $/ \mathrm{J} /$, resultado semelhante encontrado em estudo na Região Sul?.

Observamos que com relação a omissões para o fonema /z/ encontraram-se omissões de sílaba pré-tônicas e tônica em onset inicial. Não foram observadas omissões em sílaba pós-tônica indo de encontro a pesquisa realizada no Sul ${ }^{11}$, onde o único caso de omissão de sílaba encontrado envolveu a pós-tônica.

Em relação ao /z/, assim como com o /s/, foi preferencialmente substituído por/t/. Também ocorreram substituições por $/ \mathrm{f} /$, e $/ \mathrm{d} /$ resultado semeIhante encontrado em estudo na Região Sul'.

Em relação ao fonema / / /, encontraram-se omissões somente em onset inicial, corroborando com estudo realizado no Sul que relata que o onset medial é favorecedor a produção correta ${ }^{7}$. Ao que parece, a tonicidade não influenciou a presença de omissões, pois esta foi observada tanto em silaba pré-tônica como em silaba tônica, diferindo de estudo realizado no $\mathrm{Su}^{7}$ que refere que as omissões ocorrem somente em sílaba pré-tônica. Em relação à substituição, observamos que, no presente estudo, o $/ \delta /$ foi preferencialmente substituído por /t/, diferindo de estudo realizado no Sul ${ }^{7}$ onde foi preferencialmente substituído por $/ \mathrm{s} /$.

Em relação ao /3/, foi observado, no presente estudo, sua substituição por /t/. Não foi observada omissão em nenhuma posição e tonicidade. Diferindo de estudo realizado no Sul ${ }^{7}$, onde foram observadas omissões e substituições preferencialmente por $/ z /$.

Em estudo realizado no Sul ${ }^{7}$, em ordem crescente, os processos mais frequentes foram: outras substituições ([soante] e ponto), as substituições envolvendo o traço contínuo, omissões e as substituições do valor do traço [anterior]. 
No que se refere aos fonemas sonoros, é importante destacar que os achados deste estudo corroboram os da literatura nacional que afirmam que o processo de ensurdecimento não faz parte da aquisição normal, portanto, só se faz presente na fala de crianças com desvio fonológico ${ }^{10}$.

A variabilidade de resultados encontrados em pesquisas sobre a aquisição das fricativas coronais chama a atenção para outro aspecto importante a ser considerado nas pesquisas sobre a aquisição fonológica do português: a falta de rigor científico quanto aos critérios de padronização e adaptação de instrumentos ou testes para a avaliação fonológica no Brasil.

Também é importante ressaltar que, além dos aspectos linguísticos e sociais, a literatura aponta a influência de aspectos biológicos (orgânicos) sobre a aquisição fonológica ${ }^{14}$.

Portanto, diante de um fenômeno multifacetado, cabe ao fonoaudiólogo traçar ações profissionais mais rigorosas no que se refere aos parâmetros de construção dos instrumentos de avaliação fonológica.

\section{CONCLUSÃO}

A aquisição das fricativas coronais por crianças frequentadoras de escolas e creches públicas da região metropolitana do Recife sofre influência de variáveis linguísticas como ponto articulatório, posição na palavra e tonicidade e ocorre em faixa etária superior, em geral seis meses a mais, que a maioria da literatura pesquisada.

Entretanto, não há um consenso quanto às idades de surgimento ou início de produção, assim como na estabilidade ou aquisição dos segmentos, entre os estudos que objetivaram fixar parâmetros de aquisição fonológica do português brasileiro. Do mesmo modo, não há um consenso quanto aos procedimentos de avaliação fonológica utilizados nas pesquisas.

É importante salientar que há uma diversidade linguística e sócio-cultural no território brasileiro, de forma que os dados observados em algumas regiões não são necessariamente correspondentes em todas as regiões do Brasil.

Todos estes aspectos devem ser considerados em estudos que pretendem fixar parâmetros de avaliação fonológica, sobretudo se houver fins de diagnóstico de desvios fonológicos.

\section{AGRADECIMENTOS}

FACEPE; CNPq.

\begin{abstract}
Purpose: to investigate the acquisition of coronal fricatives by public county schools' children of Recife's metropolitan region. Methods: 40 children from four schools with ages between 2 and 6 year and eleven month old, whereas 20 were males and 20 females. The instrument applied for data collection was 83 images which could be referred to with 32 words carrying the phoneme target $/ \mathrm{s} /, 11$ which had the /z/ phoneme target, 21 with the / / / and 19 with the /3/; these were selected from tonicity and syllabic structure. The children were divided, according to their age, into ten different groups of four children each. The cut-point for considering an indicative of stability of the aim-phoneme was $80 \%$ of production. Results: the beginning of coronal fricatives' production took place at ages 2:0-2:5. It has been noticed that the phonemes $/ \mathrm{J} / \mathrm{,} / 3 / \mathrm{e} / \mathrm{z} /$ were acquired at ages 3:0-3:5 and the phoneme $/ \mathrm{s} /$ at ages 2:6-2:11. The phonological processes that were more frequently found are substitution and omission. We also quite often observed semantics exchange. Conclusions: children from public schools of Recife's metropolitan region have acquired the coronal fricatives later than the national literature average. It is important to highlight that there is wide linguistic and socio-cultural diversity in the Brazilian territory. For this reason data from different Brazilian regions cannot be applied homogenously in the country and therefore any attempt to determine the parameters for a phonologic evaluation ought to take this limitation into consideration, especially if such evaluation has the objective of detecting phonologic deviances.
\end{abstract}

KEYWORDS: Language Arts; Language Development; Phonetics 


\section{REFERÊNCIAS}

1. Vivar $\mathrm{P}$, León $\mathrm{H}$. Desarrollo fonológico-fonético en un grupo de niños entre 3 y 5, 11 Años. Rev. CEFAC. abr-jun 2009; 11(2):190-8.

2. Keske-Soares M, Lamprecht RR, Pagliarin KC. Aquisição não-linear durante 0 processo terapêutico. Letras de Hoje. 2008; 43:22-6.

3. Pagan LO, Wertzner HF. Análise acústica das consoantes líquidas do Português Brasileiro em crianças com e sem transtorno fonológico. Rev Soc Bras Fonoaudiol. 2007; 12(2):106-13.

4. Lamprecht RR. Sobre os desvios fonológicos. In: Lamprecht RR, Bonilha GFG, Freitas GCM, Matzenauer CLB, Mezzomo, CL Oliveira CC, et al. Aquisição fonológica do Português: perfil do desenvolvimento e subsídios para terapia. Porto Alegre: Artmed; 2004, 193-212.

5. Ferrante C, Borsel JVB, Pereira MMB. Aquisição fonológica em crianças de classe alta. Rev. CEFAC. out-dez 2008; 10(4):452-60.

6. Hora D, organizador. Estudos sociolinguísticos perfil de uma comunidade. João Pessoa: UFPB; 2004, 286p.

7. Oliveira CC. Perfil das fricativas /f/, /v/, /Š/, /Ž/ do português brasileiro. Letras de Hoje. 2003; 38(2):97-110.
8. Vi Tor RM, Cardoso-Martins C. Desenvolvimento fonológico de crianças pré-escolares da Região Noroeste de Belo Horizonte. Psicol. Rev. 2007; 13(2):383-98.

9. Keske-Soares M, Pagliarin KC, Ceron MI. Terapia fonológica considerando as variáveis linguísticas. Rev. Soc. Bras. Fonoaudiol. 2009; 14(2):261-6.

10. Hernandorena C. Aquisição fonológica do português: estabelecimento de padrões com base em traços distintivos. [tese] Porto Alegre (RS): Pontifícia Universidade Católica; 1990

11. Savio CB. Aquisição das fricativas /s/ e /z/ do Português brasileiro. Letras de Hoje. 2001; 36:721-7.

12. Bonilha GFG. Variação na aquisição fonológica uma abordagem de teoria da otimidade conexionista. Scripta. 2006; 9(18):62-76.

13. Silva CC. Aquisição da regra de assimilação de vozeamento em português brasileiro. [dissertação] São Paulo (SP): Universidade de São Paulo; 2008.

14. Lima LM, Queiroga BAM. Aquisição folológica em crianças com antecedentes de desnutrição. Rev CEFAC. 2007; 9 (1): 13-20.

DOI: 10.1590/S1516-18462011005000001

RECEBIDO EM: 01/12/2009

ACEITO EM: 07/10/2010

Endereço para correspondência:

Ana Augusta de Andrade Cordeiro

Av. Governador Agamenon Magalhães, 129 ap. 101

Recife - PE

CEP: $52030-210$

E-mail: anaaugusta_cordeiro@yahoo.com.br 